\title{
A rare side effect of Cyclophosphamide Induced Acute Interstitial Pneumonitis: a case report
}

\begin{abstract}
A 41-year-old patient with Metastatic Breast Cancer suffered from pneumonitis after administration of cyclophosphamide. A CT angiogram with IV contrast was compatible with bronchopneumonia and it was treated with broad spectrum antibiotics. Other causes of pulmonary disease were ruled out concluding patient developed cyclophosphamide induced pneumonitis. Thus, more attention is required to the serious and rare side effects of cyclophosphamide related lung toxicities. In this case report, we will focus on the rare side effects such as cyclophosphamide-induced pneumonitis (AIP) occurring in less than $1 \%$ of the population.
\end{abstract}

Volume 7 Issue 3 - 2020

\author{
Sonia Amin Thomas ${ }^{1,2}$ Harnisha Patel' \\ 'PCOM School of Pharmacy Suwanee, GA, 30024 USA \\ ${ }^{2}$ Wellstar North Fulton Hospital Roswell, GA, 30076 USA
}

Correspondence: Harnisha Patel, PCOM School of Pharmacy Suwanee, GA, 30024 USA, Email harnishapa@pcom.edu

Received: September 01, 2020 | Published: October 14, 2020
Abbreviations: FQ, fluoroquinolones; CT, computed tomography; AIP, acute interstitial Pneumonitis; ARDS, acute respiratory distress syndrome; DVT, deep vein thrombosis; LVEF, left ventricular ejection fraction; HRCT, high-resolution computed tomography; LFTs, liver function tests; IUGR, intrauterine growth restriction

\section{Introduction}

Cyclophosphamide is an alkylating chemotherapy drug that stops the cell division by cross-linking DNA strands and decreasing DNA synthesis. ${ }^{1}$ Cyclophosphamide has many side effects like most of the other chemotherapy drugs such as bone marrow suppression, hepatotoxicity, immunosuppression, cardiotoxicity, stomatitis/ mucositis, and hemorrhagic cystitis. Cyclophosphamide does not only affect cancer cells, but also healthy cells in the body. In this case report, we will focus on the rare side effects such as cyclophosphamideinduced pneumonitis (AIP) occurring in less than $1 \%$ of the population seen with cyclophosphamide. Acute interstitial pneumonitis (AIP) is identified as continuous dyspnea, dry cough, hypoxemia with lung diffuse lesions, and diffuse alveolar damage followed by the rapid development of acute respiratory distress syndrome in patients with the absence of lower respiratory lung disease that develops from days to weeks and usually requires mechanical ventilation in the majority of the patients. ${ }^{14}$ Interstitial pneumonitis is induced by the drugs that affect bronchioles, alveolar cavities, small pulmonary vessels, and further leading to pulmonary fibrosis, a more chronic cause of acute interstitial pneumonitis. The diagnostic tests include chest radiographic and high-resolution computed tomography (HRCT) often show bilateral and patchy lungs. Acute interstitial pneumonitis (AIP) cause is often unknown and most likely to be confused with acute multilobar pneumonia and many other forms of pneumonitis such as idiopathic pulmonary fibrosis, or progressive bronchiolitis obliterans-organizing pneumonia. ${ }^{3}$ The focus will be on cardiac and pulmonary toxicities such as acute interstitial pneumonitis aiming for a more clear understanding of the rare side effect that occurred after administration of cyclophosphamide.

\section{Patient case presentation}

A 41-year-old Hispanic female with an allergy to diphenhydramine (swelling) presents to the emergency department with a chief complaint of shortness of breath and abdominal pain. She reported she had severe and persistent shortness of breath, accompanied by speaking and active stomach pain, bilateral upper abdominal and lateral abdominal pain for the past two weeks with symptoms worsening. Due to her symptoms, she reports not getting sleep at night. She went through two cycles of in vitro fertilization and was about 9 weeks pregnant. She was receiving estradiol and progesterone treatment by her fertility doctor and was frequently seen by her obstetrician for regular checkups. She had a past medical history of gas/reflux and indigestion. She had a family history of colon and breast cancer. She did not smoke cigarettes or drink alcohol. She reported changes in both breasts such as an increase in size and right breast changes and skin discoloration.

Hallmarks of widely disseminated bone metastasis, osteolytic to several vertebrae, as well as very extensive hepatic metastasis were present. Large tumor process on the right breast with "green-mild peu d'oreange" axillary lymphadenopathy. An ultrasound-guided biopsy was performed, and pathological results were reported as aggressive breast cancer, with high mitotic index (ER: 90\%, PR: 90\%) positive, and HER 2 positive. She was diagnosed with stage IV advanced metastatic inflammatory breast cancer of the right breast. Her physical examination noted scleral icterus and laboratory findings were abnormal for calcium being high, bilirubin being low, and abnormally high liver function tests, proBNP levels were high, and CBC (mainly WBC, platelets and hgb). Since her tumor progressed to the other sites of the organ such as liver and bone she was diagnosed with malignant hypercalcemia. She was treated with zoledronic acid $4 \mathrm{mg}$ IV every 4 weeks with calcium and vitamin D daily as she had a normal renal function and dexamethasone $4 \mathrm{mg}$ by mouth twice daily in addition to furosemide $20 \mathrm{mg}$ by mouth twice daily and spironolactone $25 \mathrm{mg}$ by mouth twice daily for anasarca due to hypoalbuminemia and increase proBNP levels. She had very limited chemotherapy options due to her pregnancy and since she was in her first trimester systemic therapy was not an option until her second trimester.

She underwent dilation and curettage surgery and interrupted her pregnancy so she can be started on her systemic therapy as soon as possible. She was depressed after her surgery and was started on a sertraline $50 \mathrm{mg}$ tablet by mouth once daily. Docetaxel was not an option for her chemotherapy due to an increase in the hepatic metastasis throughout the liver with jaundice and abnormal LFTs so she was started on pertuzumab, trastuzumab, and cyclophosphamide as a 
systemic chemotherapy option. She was also prescribed ondansetron, prochlorperazine, and olanzapine for nausea prevention, and kneehigh ted hose for deep vein thrombosis (DVT) prophylaxis. Gradual improvement was seen overall with her abdominal pain, jaundice, swelling, and anasarca. However, after two weeks, she had a very intense sudden onset of knee pain possibly gout or pseudogout for which she was started on indomethacin $50 \mathrm{mg}$ by mouth twice daily with meals. Echocardiogram was performed and the left ventricular ejection fraction (LVEF) was over $60 \%$. Also, a computed tomography (CT) scan was performed notable for lower lobe pneumonia for which she was prescribed levofloxacin $750 \mathrm{mg}$ by mouth every day for 7 days.

She received her second cycle of chemotherapy after three weeks and decadron was discontinued. After receiving two cycles of cyclophosphamide, pertuzumab, and trastuzumab two weeks later, she again presents to the emergency department with a chief complaint of shortness of breath and exertional cough despite antibiotic therapy. Later, a chest X-ray revealed bilateral pneumonia. Her blood cultures were drawn, but came back negative twice and so was started on broadspectrum antibiotic therapy. She received one dose of azithromycin $500 \mathrm{mg} \mathrm{IV}$ once at $250 \mathrm{ml} / \mathrm{hr}$ over 60 minutes and ceftriaxone $2 \mathrm{~g}$ IV once for bronchopneumonia. She developed mouth sores due to cyclophosphamide for which she was prescribed acyclovir 5\% ointment. The primary concern was to see an improvement in her respiratory illness so her chemotherapy treatment was delayed until she was stable. Later, after a week she was chronically ill, fatigue, weak, hypoxic, and tachycardic. Her chest X-ray revealed bilateral interstitial and airspace infiltrates most prominently in the left upper and right lower lobe. She was started on broad-spectrum antibiotics such as zosyn $3.375 \mathrm{~g} \mathrm{IV}$ every 8 hours and doxycycline $100 \mathrm{mg}$ IV every 12 hours and was placed on a high flow of nasal cannula.

Due to the need for oxygen, she was not a candidate for BAL. Meanwhile, improving on LFTs, shortness of breath, and tachycardia was noticed. She was due for her third cycle of chemotherapy, but it was put on hold as the major concern was her respiratory illness. She was clinically improving without any pain, but still had crackles and rhonchi. Her cyclophosphamide therapy was put on hold, but as she was significantly improving she was only given anti-HER2 therapy. Later, the gradual and steady improvement was seen with her total bilirubin and LFTs, shortness of breath and was afebrile.

For ARDS, she almost needed mechanical ventilation, but it was avoided due to aggressive medication therapy such as broad-spectrum antibiotics and empiric steroids. Once she was stable, she received only anti-HER 2 therapy, shortly after receiving anti-HER2 therapy she developed chills and shivers that were resolved with dexamethasone. It was confirmed that she had cyclophosphamide-induced pneumonitis. Later on, her hepatitis functions were normal; thus she was switched to carboplatin/docetaxel, perjeta, and trastuzumab for the rest of the treatment hence no cytotoxic myelosuppression chemotherapy was given.

Table I Laboratory findings

\begin{tabular}{|c|c|c|c|c|c|}
\hline Labs & $03 / 27$ & $03 / 28$ & $05 / 09$ & $05 / 13$ & $05 / 18$ \\
\hline WBC & $18.6 \mid$ & 12.66 & 5.63 & 6.90 & 6.01 \\
\hline $\mathrm{Hgb}$ & 11.10 & 9.80 & 10.0 & 8.50 & 8.70 \\
\hline Total calcium & 13.30 & 12.70 & 8.5 & 8.3 & 8.90 \\
\hline AST & 848 & 760 & 125 & 85 & 45 \\
\hline Bilirubin & 3.6 & 2.90 & 2.2 & 1.7 & 1.30 \\
\hline Scr & 0.47 & 0.42 & 0.43 & 0.42 & 0.34 \\
\hline $\mathrm{Hct}$ & 33.70 & 29.90 & 32.0 & 26.4 & 28.10 \\
\hline Sodium & $|3|$ & 135 & 134 & 136 & 137 \\
\hline ALT & 266 & 237 & 176 & 108 & 47 \\
\hline S.Albumin & 3.0 & 2.6 & 3.4 & 2.6 & 2.50 \\
\hline $\mathrm{CO} 2$ & 20 & 22 & 22 & 21 & 22 \\
\hline ALK phos & 662 & 605 & 782 & 613 & 481 \\
\hline Platelets & 260 & 237 & 259 & 243 & 385 \\
\hline T. protein & 6.0 & 5.4 & 6.8 & 5.9 & 6.30 \\
\hline \multirow[t]{2}{*}{ proBNP } & 402 & - & - & - & 2456 \\
\hline & & & & & $2.10(1410)$ \\
\hline Lactic Acid & - & - & - & - & $1.5(1520)$ \\
\hline CRP Quant & - & - & - & - & 12.10 \\
\hline
\end{tabular}




\section{Discussion}

Many antineoplastic drugs can cause undesired pulmonary toxicities. Cyclophosphamide is one of the widely used drugs for chemotherapy treatment. Cyclophosphamide induced pneumonitis can be acute or chronic, and can further lead into pulmonary fibrosis, pulmonary edema, and etc ${ }^{13}$ Cyclophosphamide induced lung toxicities are hard to differentiate due to the presence of the confounding variables, for example, radiation pneumonitis, opportunistic infection, use of other cytotoxic drugs, oxygen toxicities, and pulmonary malignancies. ${ }^{8}$ Moreover, diagnosis was not made only based on symptoms, but also looking at the length of use of cyclophosphamide in the treatment, chest imaging findings, and nonappearance of other toxicities. ${ }^{12}$ However, other pulmonary diseases were ruled out, confirming that the patient developed cyclophosphamide induced pneumonitis.

The cyclophosphamide manufacturing label reports pneumonitis as a rare condition associated with cyclophosphamide. ${ }^{1,2}$ Even though cyclophosphamide is metabolized in the liver, it is metabolized in its active metabolite, acrolein, in the lungs. ${ }^{5}$ Acrolein causes peri oxidative injury by developing alterations in the physical state of the lipid bilayer being the major cause of inactivation of membrane bound receptors. ${ }^{5}$

Lung toxicity is differentiated into early and late onset of pneumonitis. Early onset of pneumonitis is often reversible. The most common symptoms seen are cough and dyspnea within 6 months of receiving cyclophosphamide, but early onset is often treatable with corticosteroids after discontinuation of cyclophosphamide. ${ }^{12}$ However, late onset of pneumonitis is irreversible and mostly unresponsive to corticosteroids. Bilateral pleural thickening is most commonly seen with late onset pneumonitis. The cause remains idiopathic, but late onset has a chronically progressive course for the treatment. ${ }^{8}$

Even though, cyclophosphamide induced pneumonitis is a rare condition occurring in less than one percent of the population, other forms of pulmonary include pneumonia, subacute interstitial lung disease, acute respiratory distress syndrome, and pulmonary edema are described as rare conditions with cyclophosphamide as well. ${ }^{4}$ The trials comparing frequency of toxicities associated with different chemotherapy regimens for hematological and solid tumors differ within $21.4 \%$ and $58 \%{ }^{5,6,9}$ However, in all the trials another chemotherapy regimen, radiation therapy, or surgery could have been involved. No major lung toxicity was reported after receiving cyclophosphamide dose of $2,000 \mathrm{mg} / \mathrm{m} 2$ in children after 18 year of follow up. ${ }^{10}$

The cyclophosphamide has shown teratogenic effects in humans by promoting Intrauterine growth restriction (IUGR), deformity of head and extremities such as ocular involvement, craniostenosis, facial dysmorphia, hydro or micro cephalis. ${ }^{7}$ It is hard to find dose dependent relationships as there are very rare cases reported. ${ }^{7}$ Some malformations observed with the low dose seem to be secondary to exposure during the first trimester, while no teratogenic effects were noticed with the high dose of cyclophosphamide in the new borns. ${ }^{7}$

Hematological effects seen in newborns were during the second and third trimester, concluding that it is absolutely contraindicated during pregnancy. However, if the use is absolutely necessary during pregnancy, for example, breast cancer then it should be recommended after the first trimester if it can be done. ${ }^{7}$ The use of contraceptives should be carried on through the end of treatment or at least minimum of one ovulation cycle after the end of the treatment before considering fertilization.

In concise, the occurrence of cyclophosphamide induced pneumonitis is very rare, but when alveolar and interstitial damage occurs, progression to pulmonary fibrosis is poor as it cannot be stopped with the use of steroids and is often quick resulting in poor prognosis. ${ }^{4}$

\section{Conclusion}

This case report corresponds to the use of cyclophosphamide as a highly probable causative agent of pneumonitis in this patient. Given that other pulmonary causes were ruled out in this case, early onset of the pneumonitis in the relationship was seen with cyclophosphamide. Cyclophosphamide was the one causing pulmonary disease. As late onset causes remain idiopathic it is really hard to differentiate the pneumonitis cause related to cyclophosphamide in each patient as everyone's body reacts differently with drugs. The high dose of cyclophosphamide has not been developed to be a risk factor; however, with this patient she developed pneumonia after a week of receiving a high dose of cyclophosphamide further leading into ARDS. ARDS was prevented with aggressive medication treatment. This case helps to assert the importance in evaluating cyclophosphamide use as a potential cause of pneumonitis.

\section{Conflicts of interest}

The authors stated that they had no conflict of interest.

\section{Funding}

No financial support was received to the authors for the research, publication, and authorship.

\section{References}

1. Cyclophosphamide FDA Label. USA: Baxter Company; 2020.

2. Cyclophosphamide Powder Baxter Company. USA. 2020.

3. Bonaccorsi A, Cancellieri A, Chilosi M, et al. Acute interstitial pneumonia: report of a series. Eur Respir J. 2003;21(1):187-191.

4. Brieva J. Cyclophosphamide-induced acute respiratory distress syndrome. Respirology. 2007;12(5):769-773.

5. Brockstein BE, Smiley C, Al-Sadir J, et al. Cardiac and pulmonary toxicity in patients undergoing high-dose chemotherapy for lymphoma and breast cancer: prognostic factors. Bone Marrow Transplant. 2000;25(8):885894.

6. Chap L, Shpiner R, Levine M, et al. Pulmonary toxicity of high-dose chemotherapy for breast cancer: a non-invasive approach to diagnosis and treatment. Bone Marrow Transplant. 1997;20(12):1063-1067.

7. Leroy C, Rigot J, Leroy M, et al. Immunosuppressive drugs and fertility. Orphanet J Rare Dis. 2015;10:136.

8. Malik SW, Myers JL, DeRemee RA, et al. Lung toxicity associated with cyclophosphamide use. Two distinct patterns. Am J Respir Crit Care Med. 1996;154(6 Pt 1):1851-1856.

9. Morgan M, Dodds A, Atkinson K, et al. The toxicity of busulphan and cyclophosphamide as the preparative regimen for bone marrow transplantation. Br J Haematol. 1991;77(4):529-534. 
10. Morse CC, Sigler C, Lock S, et al. Pulmonary toxicity of cyclophosphamide: a 1-year study. Exp Mol Pathol. 1985;42(2):251-260.

11. Patel JM. Metabolism and pulmonary toxicity of cyclophosphamide. Pharmacol Ther. 1990;47(1):137-146.

12. Pugh D, Farrah TE, Gallacher PJ, et al. Cyclophosphamide-Induced Lung Injury. Kidney Int Rep. 2018;4(3):484-486.
13. Qian $\mathrm{P}$, Peng $\mathrm{CH}$, Ye $\mathrm{X}$. Interstitial pneumonia induced by cyclophosphamide: A case report and review of the literature. Respir Med Case Rep. 2019;26:212-214.

14. Raissy HH, Harkins M. Drug-Induced Pulmonary Diseases. In: DiPiro JT, Yee GC, Posey L, et al. editors. Pharmacotherapy: A Pathophysiologic Approach, 11e.USA, McGraw-Hill: Access Pharmacy; 2020. 